\section{Ocular injuries from fireworks: the 11-year experience of a US level I trauma center}

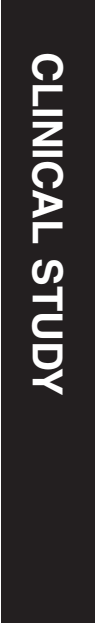

${ }^{1}$ Division of Oculoplastic Surgery, Department of Ophthalmology, University of Washington School of Medicine, Seattle, WA 98104, USA

${ }^{2}$ Department of Ophthalmology, Southern California Permanente Medical Group, San Diego, CA 92120, USA

Correspondence: SS Shaftel, Department of Ophthalmology, Southern California Permanente Medical Group, 4405 Vandever Avenue, San Diego, CA 92120, USA Tel: +1 (844) 424 1867; Fax: +1 (619) 5167181

E-mail: sshaftel@uw.edu

Received: 18 October 2015 Accepted in revised form: 7 April 2016 Published online: 10 June 2016

\begin{abstract}
Purpose Characterize ocular trauma and visual outcomes from firework injuries at a level I trauma center.

Methods Retrospective review of all firework injuries at Harborview Medical Center between 2003 and 2013.

Results Three hundred and twenty-seven patients sustained firework injuries, of which 100 (31\%) sustained ocular injuries. The average age of all patients who sustained fireworks injuries was 24.2 years, $89 \%$ were male and $54 \%$ of injuries occurred within $48 \mathrm{~h}$ of $4 \mathrm{July}$. Ocular injuries were most commonly caused by mortars $(24 \%)$ and rockets $(22 \%)$. Rockets were associated with four times the frequency of ocular injuries as compared with non-ocular injuries $(P<0.001)$. Spectators were more likely to sustain ocular injuries than non-ocular injuries $(P=0.001)$. The most common injuries sustained were corneal abrasions (67\%), hyphemas (42\%), eyelid injuries $(39 \%)$, and ruptured globes $(17 \%)$. Twentyeight percent of patients with ocular injuries required surgical intervention. Ruptured globes occurred in $17 \%$ of patients, with the majority being complex corneoscleral lacerations. Fifty-eight percent of patients who sustained ruptured globes were left with no light perception in the affected eye. Average follow-up was 188 days. Average visual acuity significantly improved from $\log$ MAR $1.8 \pm 1.6$ at presentation to $\log$ MAR $1.3 \pm 1.8$ at last follow-up.

Conclusions Firework-related ocular trauma frequently results in vision-threatening pathology. Prompt referral to and treatment by ophthalmologists is critical. This study documents the dangers inherent in the personal use of fireworks and provides data that may help guide public policy to decrease the frequency of these devastating injuries. Eye (2016) 30, 1324-1330; doi:10.1038/eye.2016.104; published online 10 June 2016
\end{abstract}

IT Chang ${ }^{1}$, MA Prendes ${ }^{1}$, KJ Tarbet ${ }^{1}$, AJ Amadi ${ }^{1}$, S-H Chang ${ }^{1}$ and SS Shaftel ${ }^{2}$

\section{Introduction}

Fireworks are used worldwide for festive purposes and are particularly popular in the United States during celebrations for 4 July and New Year's Eve. Unfortunately, fireworks have been associated with various bodily injuries, permanent disabilities, and even death. ${ }^{1-4}$ In 2013 alone, an estimated 11400 firework-related injuries were treated in US hospitals, with $65 \%$ occurring in the 30 days surrounding 4 July. A significant portion (16\%) of these fireworkrelated injuries were ocular injuries. ${ }^{1}$

Firework-related ocular trauma is particularly devastating because the majority of these injuries occur in young adults and often result in lifelong disability secondary to severe vision loss. ${ }^{5-9}$ Given the substantial economic costs to society and their preventable nature, firework-related ocular injuries are a serious public health concern. ${ }^{4-6,10}$ Current precise data regarding the types of injuries and outcomes of patients affected by eye injuries from fireworks are lacking in the United States, but has been reported in other countries. ${ }^{2,5-8,11-18}$

The purpose of this study is to better characterize the clinical features, outcomes, and management patterns of ocular trauma from fireworks in the United States through the creation of the most comprehensive data set published to date. We performed a retrospective chart review of 327 patients presenting to our level I trauma center over an 11-year period. By documenting the scope of ocular injuries in the United States, we hope to promote awareness of the dangers that fireworks pose to the eye, and help guide public policies aimed at reducing the number of firework-related ocular injuries.

Methods

Approval for this retrospective chart review was received from the University of Washington Institutional Review Board, and this study has 
been Health Insurance Portability and Accountability Act-compliant. Subjects were identified in the University of Washington trauma registry for Harborview Medical Center. Harborview is the sole level I adult and pediatric trauma and burn center serving the five states of Washington, Wyoming, Alaska, Montana, and Idaho.

The trauma registry includes patients with the following ICD-9 external cause of injury codes (E-codes): 800-904 or 910-959 or individual codes 994.1, 994.7, 994.8, 991.6, and 991.3. In addition, any of the following trauma patient criteria allow for their inclusion in the registry: activation of trauma resuscitation team, 'Dead on Arrival', death in the emergency department or in the facility, transfers to or from the emergency room and another facility via ambulance, patients flown from the scene, and all gunshot wounds.

The inclusion data for this study were as follows: patients registered in the trauma database between 1 January 2003 and 31 December 2013, use of ICD-9 E-codes 923.0 or 923.1 (accident caused by fireworks or blasting materials, respectively), and presence of the trauma registry cause code-explosion. The data manager of the trauma registry supplied an initial list of 467 patients satisfying these conditions. The electronic medical records of these patients were reviewed and pared down to 327 injuries caused by fireworks. Data were then collected with regard to the following variables: age, sex, injuries sustained, type of firework, location at the time of injury, alcohol use, ocular injuries sustained, transfer status, medical specialty performing repair, surgical interventions, location where repair was performed, visual acuity at the time of injury, visual acuity at last follow-up, past ocular history, presence of associated infection, and length of follow-up. Snellen visual acuity was converted to $\log$ MAR acuity for data analysis. For those with $\log$ MAR $>1.68$, recording was as follows: count fingers $(\log M A R 2)$, hand motion $(\log M A R \quad 3)$, light perception (logMAR 4), and no light perception $\left(\log\right.$ MAR 5). ${ }^{19}$

The data were de-identified after the chart review was completed, and data analyses were performed in
Microsoft Excel 2007. Graphs and figures were created using GraphPad Prism 6 (Graphpad Software, San Diego, CA, USA). Variability is listed as s.e.m. For the purposes of this study, children were defined as patients less than 18 years of age at the time of injury. Significance was determined using a Student's two-tailed $t$-test when comparing intake and final visual acuity, with significance set at $P \leq 0.05$. $X^{2}$-analysis was used when comparing frequencies of variables present between separate groups and a resulting odds ratio (OR) generated. All percentages were rounded to the nearest whole number for presentation purposes.

\section{Results}

Patients who sustained firework injuries between 2003 and 2013 were identified in the Harborview Medical Center trauma registry using E-codes 923.0 or 923.1 while limiting the etiology to those inflicted by explosions. Out of 467 patients identified, 327 had firework injuries after detailed chart review was performed to eliminate other etiologies (Table 1). The average age of these patients was 24.2 years ( \pm 13.2 years), with $37 \%$ being less than 18 years old. Male patients accounted for $89 \%$ of cases, whereas only $11 \%$ were female. The data sets were then divided into patients sustaining or not-sustaining ocular injuries. One hundred patients (31\%) sustained ocular injuries, whereas 227 (69\%) did not. The sex distribution was similar among the two groups, with $89 \%$ being male and $11 \%$ female. The average age of patients with ocular injuries was not significantly different from those without ocular injury (24.0 years \pm 14.0 vs 24.3 years \pm 13.0 , $P=0.85)$. Fifty-three percent of all injuries (54\% of ocular injuries) occurred within $48 \mathrm{~h}$ of 4 July, and $6 \%$ (10\% of ocular injuries) occurred within $48 \mathrm{~h}$ of New Year's Eve.

The initial analysis looked at all types of firework injuries identified in the trauma registry. Mortar-type fireworks were the most common cause of firework injuries, being documented in $23 \%$ of all patients (Supplementary Table 1). Other types of fireworks responsible for more than $3 \%$ of all injuries were crackers

Table 1 Patient demographics

\begin{tabular}{lccc}
\hline & All firework injuries & Ocular firework injuries & Non-ocular firework injuries \\
\hline Number of patients & 327 & 100 & 227 \\
Age (mean \pm SD) & $24.2 \pm 13.2$ & $24.0 \pm 14.0$ & $24.3 \pm 13.0$ \\
$\%$ Younger than 18 & $37 \%$ & $41 \%$ & $35 \%$ \\
Male : female & $290: 37(89 \% \mathrm{M})$ & $89: 11(89 \% \mathrm{M})$ & $201: 26(89 \% \mathrm{M})$ \\
Confirmed alcohol use during accident & $19 \%$ & $16 \%$ & $20 \%$ \\
$\%$ Injuries occurring within 48 h of New Year's Eve & $6 \%$ & $10 \%$ & $4 \%$ \\
$\%$ injuries occurring within 48 h of 4 July & $53 \%$ & $54 \%$ & $53 \%$ \\
\hline
\end{tabular}

Abbreviation: M, male.

The first column shows the data for all patients presenting after firework injuries. This group is then subdivided into those patients with ocular injuries and those without ocular injuries. 
(19\%), homemade explosives $(14 \%)$, rockets $(10 \%)$, sparklers $(8 \%)$, and roman candles $(6 \%)$. The type of firework involved was unknown in $16 \%$ of cases. For the subset of patients with ocular injuries, mortar-type fireworks were the most common cause of firework injury, being documented in $24 \%$ of patients, followed by rockets in $22 \%$ (Figure 1). Other types of fireworks responsible for more than $3 \%$ of ocular injuries were homemade explosives (14\%), firecrackers $(10 \%)$, roman candles $(8 \%)$, sparklers $(5 \%)$, and gunpowder $(3 \%)$. The type of firework was unknown in $12 \%$ of patients with ocular injuries. Interestingly, rockets were associated with four times the frequency of ocular injuries (22\%) as compared with non-ocular injuries $(5 \%$, OR $4.9, P<0.001)$. Patients younger than age 18 were more likely to sustain injuries from rockets, gunpowder and crackers, whereas patients older than age 18 were more likely to sustain

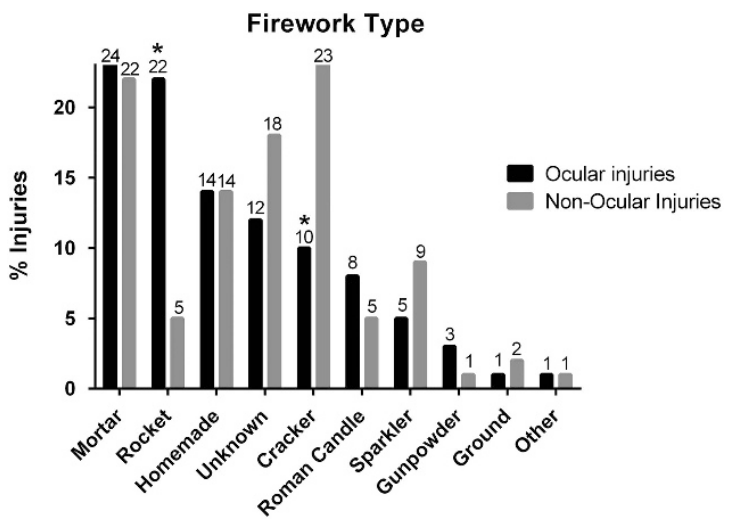

Figure 1 Percentage of injuries by firework type. The percentage of patients with ocular injuries and without ocular injuries are graphed separately by each type of firework. *signifies a significant difference between groups with respect to firework type $(P<0.05)$.

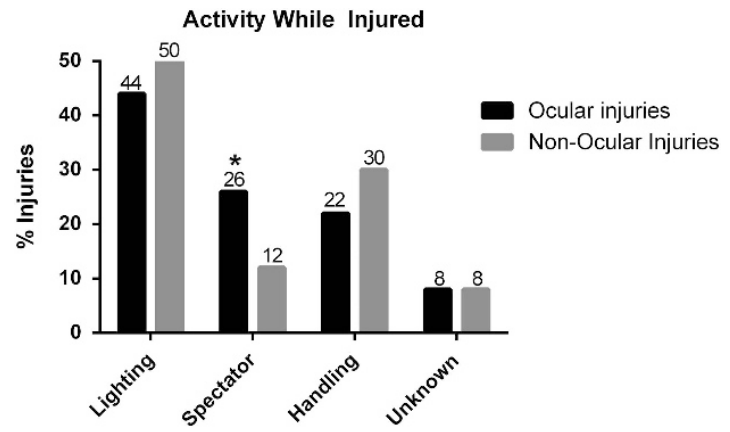

Figure 2 Percentage of injuries by activity. The percentage of patients with ocular injuries and those without ocular injuries are graphed separately by each type of activity. *signifies a significant difference between groups with respect to activity $(P<0.05)$. injuries from mortar, sparkler and homemade fireworks $(P<0.001$, Supplementary Table 2$)$.

Activities associated with all injuries are listed in Supplementary Table 1. Activities associated with ocular injuries were lighting (44\%), spectator $(26 \%)$, handling $(22 \%)$, and unknown $(8 \%$, Figure 2$)$. Spectators were involved in a much larger proportion of the ocular injuries $(26 \%)$ compared with non-ocular injuries $(12 \%$, OR 2.6, $P<0.001)$. Only one $(1 \%)$ of the ocular injuries was sustained during a professional firework display; in this case the patient was also handling the device.

Documented alcohol use was associated with $19 \%$ of all injuries, with no significant difference between ocular injuries $(16 \%)$ vs non-ocular injuries $(20 \%, P=0.44)$. However, alcohol use was undocumented in $43 \%$ of patients within each group.

Further analysis focused on the subgroup of patients who sustained ocular injuries from fireworks. A variety of ocular injuries resulted from fireworks, with frequencies as noted in Figure 3. Corneal abrasions were most frequent and occurred bilaterally in $8 \%$ of patients, whereas $1 \%$ of patients had bilateral hyphema.

Chorioretinal injuries included commotio retinae choroidal rupture, and intra-retinal hemorrhage. Traumatic glaucoma was secondary to iridodialysis or angle recession. Hyphema was strongly associated with the use of rockets $(73 \%$ of rocket injuries resulted in hyphema) and crackers $(50 \%, P=0.007)$. Eyelid laceration was associated with patients more than 18 years old; other types of injuries were not found to be associated with age of the patient (Supplementary Table 2).

Ruptured globes presented in 17 patients (17\%), with one patient sustaining a bilateral rupture. The majority of

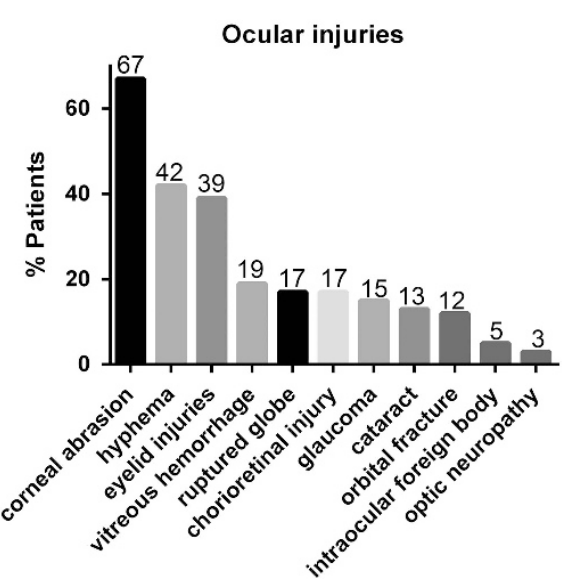

Figure 3 Ocular injuries resulting from fireworks. The types of ocular injury seen in this study are graphed as a percentage of patients affected with values listed above each corresponding bar. Of the $39 \%$ of patients with eyelid injuries, $26 \%$ had margininvolving lacerations and $8 \%$ had canalicular lacerations. 


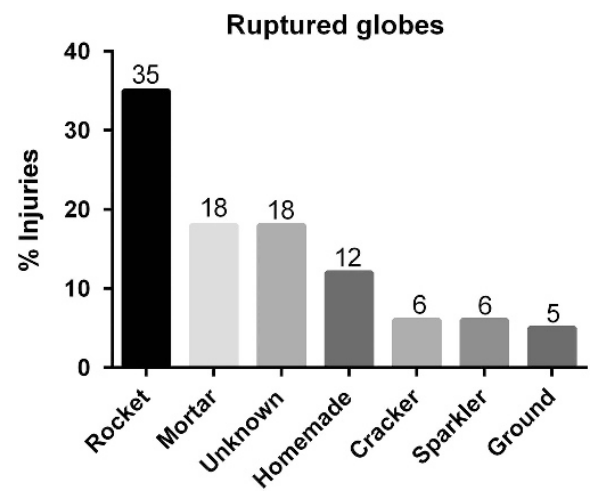

Figure 4 Ruptured globes by firework type. Seventeen patients suffered a ruptured globe during our study period. Firework type is displayed with the percentage of rupture globes they caused with values listed above each corresponding bar.

ruptured globes injuries were full-thickness lacerations involving both the cornea and sclera (eight cases).

There were six cases of complex corneal lacerations or avulsions, three isolated scleral lacerations, and one severely deformed globe without recognizable ocular structures. Among the ruptured globes, there were five cases of intraocular foreign body (IOFB). Two cases of IOFB were limited to the anterior chamber, and three cases had IOFB present in the posterior cavity. The ruptured globe injuries most commonly resulted from rockets with the percentage of each firework type noted in Figure 4 . The activities of those who sustained ruptured globes were lighting (35\%), spectator $(35 \%)$, handling $(18 \%)$, or unknown $(12 \%)$. Notably, $67 \%$ of the rocket and $100 \%$ of the mortar injuries leading to globe rupture necessitated eventual enucleation.

The average follow-up period was 188 days (6 months) with a range of 1-2588 days. Follow-up was established in $81 \%$ of patients and was limited in many patients by extreme travel distances and transfer of care to other practitioners upon discharge. Seventy-three percent of patients were transferred from other hospitals.

Twenty-eight of the hundred ocular injury patients $(28 \%)$ required surgical intervention in the operating room, and the average number of surgeries for patients who required surgery was 1.9 (range 1-7). The surgeries performed were ruptured globe exploration and repair (17 patients, one was bilateral), enucleation (10 patients, one was primary enucleation for unrepairable globe), plastics reconstruction (10 patients), posterior segment surgeries (12 patients), and anterior segment repair (five patients). The plastics reconstructive surgeries included eyelid laceration repair (eight patients), enucleation with or without socket reconstruction (10 patients), dermis fat grafting (two patients), cicatricial ectropion and entropion repair (two patients), ocular surface reconstruction with symblepharon repair and mucous membrane grafting (one patient), and orbital wall fracture repair (one patient). Three patients required more than five surgeries. One patient sustained bilateral ruptured globes from homemade fireworks and eventually required a total of seven surgeries including tectonic penetrating keratoplasty, multiple vitrectomies, and glaucoma drainage device placement with a final visual acuity of light perception and 20/70. Another patient sustained a catastrophic ruptured globe from a bottle rocket requiring primary enucleation, with five subsequent oculoplastics procedures for dermis fat grafting, socket reconstruction, and eyelid repositioning. The third patient underwent ruptured globe repair and five retinal surgeries for recurrent retinal detachment and proliferative vitreoretinopathy and had a final visual acuity of $5 / 200$. In addition to those requiring repair in the operating room, patients also underwent bedside procedures performed under local anesthesia such as corneal or conjunctival foreign body removal (two patients) and eyelid laceration repair (four patients). The 28 patients who required operative repair only include those who were taken to the operating room by the ophthalmology service, and does not include other ocular or orbital injuries such as complex eyelid lacerations and orbital fractures that may have been repaired by other services.

Initial visual acuity ranged from $20 / 20$ to no light perception, and was documented in 86 patients, with an average $\log$ MAR of $1.7 \pm 1.6$ (98 eyes, Snellen visual acuity of $\sim 20 / 1000$ ). Fourteen patients were not included in this calculation: nine patients were unable to be assessed because of altered mental status and five were pediatric patients with visual acuity recorded as fix and follow. The best-corrected visual acuity at last follow-up was $\log$ MAR $1.3 \pm 1.8$ in 73 patients (82 eyes, Snellen visual acuity of $\sim 20 / 400$ ). Three of these patients had light perception, 11 no light perception, and 27 patients were not included in the calculation because of fix and follow visual acuity (three patients) or lack of documentation or follow-up (24 patients). For the patients with measurable visual acuity both at presentation and last follow-up, there was a significant improvement of visual acuity from $\log$ MAR $1.8( \pm 1.6$, Snellen VA $\sim 20 / 1300)$ to $\log$ MAR $1.3( \pm 1.8$, Snellen VA $\sim 20 / 400)$, respectively (77 eyes, $P<0.05$ ). Six out of twenty-six spectators $(23 \%)$ who sustained ocular injuries had ruptured globes, with a final visual acuity of light perception or worse in the affected eye. Of the 18 globe ruptures (17 patients), 10 (59\%) of these eyes were left with no light perception at last follow-up. The five patients with intraocular foreign bodies had a final visual acuity of $\log$ MAR $2.2( \pm 1.9$, Snellen visual acuity of $\sim 20 / 3300$ ). 


\section{Discussion}

Fireworks are pyrotechnic devices commonly displayed worldwide during festive occasions such as celebrations of national and religious events. As such, firework-related injuries are common around the time of these occasions, such as Diwali in India, the Spring festival in China and Aidil Fitri celebration in Malaysia. ${ }^{13,14,17}$ In the United States, consumers are estimated to spend over $\$ 600$ million dollars on fireworks during 4 July each year. ${ }^{20}$ Given the highly incendiary and explosive nature of fireworks, their use is often associated with serious injury and cost to society. In 2011 alone, fireworks cost an estimated \$32 million dollars of direct property damage in the United States. ${ }^{21}$ In addition, the cost of care related to firework injuries and ocular trauma is substantial. A previous study estimated that the federal cost of blindness is approximately $\$ 11896$ per person year for a working-aged American related to reduced potential earnings, assistance programs, and tax losses. ${ }^{22}$ As firework injury disproportionately affects a younger population with devastating injuries, the cost to society is substantial.

Although high-powered display fireworks are restricted by federal law in the United States, consumergrade fireworks are widely available and legal in most states. ${ }^{23}$ Despite their legality, they remain extremely dangerous and are frequently associated with significant morbidity and even mortality. ${ }^{1,4-7,11,12,24}$ The most commonly injured body parts are hands, eyes, head, and face. ${ }^{1,4}$ Not surprisingly, ophthalmologists are frequently involved in the care of these patients because of the high rates of ocular injury. ${ }^{5-8,12,13,18,25}$ This study represents one of the most comprehensive data sets to date describing ocular injuries from fireworks in the United States, and serves to update findings last published over 10 years ago in this country. ${ }^{8,18,26}$ Previous studies have focused on the incidence of ocular trauma from fireworks in the United States, but have neglected reporting of outcomes, morbidity, and extent of vision loss. 1,8,18,26 Similar international studies have addressed these issues, but the regulation of specific firework devices outside the United States is variable, making extrapolation of the data to the US population difficult. $5,6,8,9,11,12,27$

Most of the ocular injuries in this study occurred within $48 \mathrm{~h}$ of 4 July (US Independence Day) and New Year's Eve, together accounting for $64 \%$ of all ocular injuries over 11 years. Eye trauma occurred in $31 \%$ of the patients with general firework trauma, which is slightly higher than the previously reported rate of $17-22 \% .1,5$ Similar to prior studies, the majority of firework-related ocular injuries affected young males, an average age of 24 years, and a significant proportion (35\%) of all ocular injuries occurred in children. ${ }^{1,5-9,11-14,18,24,28}$
The ocular injuries varied in presentation and severity. A significant proportion of these injuries resulted in permanent vision loss, with the mean Snellen visual acuity of $\sim 20 / 1000$ at presentation. Intervention led to a significant improvement in visual acuity, and at last follow-up the mean Snellen visual acuity was $\sim 20 / 400$. Factors that have been associated with poor visual outcome in other publications included open globe injury, poor initial visual acuity, IOFB, retinal detachment, and development of endophthalmitis.9,12,16 In our study, open globe injury and IOFB were indeed often associated with very poor visual outcome. Fifty-eight percent of open globe injuries resulted in no light perception vision, and IOFB injuries led to an average final Snellen visual acuity of $\sim 20 / 3300$.

The most common injuries were corneal abrasions, hyphemas, and various eyelid injuries (Figure 3). Ruptured globes occurred in $17 \%$ of patients, which is similar to previously reported rates of $7-19 \%$. ${ }^{7,8,12,13}$ Mortars, rockets, and homemade fireworks were most commonly associated with ocular injuries (Figure 1). Although there is no known association between the detonation power of the pyrotechnic and the severity of injury, we found that injuries from mortar, rockets, and homemade fireworks were not only the most frequent but were usually severe, accounting for $65 \%$ of ruptured globe cases including one bilateral ruptured globe from homemade fireworks. ${ }^{6}$ Two-thirds of the open globe injuries from rockets and all of the open globes from mortar fireworks eventually required enucleation, highlighting the complexity and severity of these injuries.

A significant proportion of patients required surgical intervention $(28 \%)$. These patients averaged 1.9 trips to the operating room. The most frequently performed surgeries were ruptured globe repair, enucleation, retinal detachment repair, plastics and ocular surface reconstruction, glaucoma drainage device placement, penetrating keratoplasty, and traumatic cataract extraction. Comprehensive management with early involvement of multiple ophthalmic subspecialties is critical in the care of these patients, given the breadth and complexity of procedures utilized in their care.

Ocular injuries from professional firework displays are relatively rare. ${ }^{5}$ Indeed, only one patient in our study sustained ocular injury during a public firework display, and the patient was the one who was handling the firework. Similar to previous studies, $99 \%$ of the injuries in our study occurred from consumer-grade and homemade fireworks. ${ }^{2,6,7,11}$ An important observation of this study is that injuries occur frequently in spectators. In our study, $26 \%$ of ocular injuries occurred in bystanders, and $23 \%$ of these patients had ruptured globes. Given the frequency and severity of injury from consumer-grade and homemade fireworks documented in this study, 
we agree that there should be strict and restrictive laws on personal use of fireworks not only for the safety of those handling the fireworks but also for spectators. 8,18,27,29

Four states in the United States have banned the sale of all fireworks, and these states have a lower rate of fireworkrelated injuries. $5,18,23,26,28$ It has been well established that regions where all fireworks are banned, except those used for public display, have a lower incidence of fireworkrelated ocular injury. $5,8,18,26$ In a recent systematic review of data from multiple different countries, the mean injury rate was estimated at 2.70/100 000 in regions with permissive firework laws concerning private firework use compared with $0.35 / 100000$ in regions with restrictive firework laws, representing an $87 \%$ decrease in ophthalmic firework trauma in regions with restrictive laws. ${ }^{5}$ This would translate to 87 fewer patients with ocular injuries in our study, 14.8 fewer ruptured globes, and 9.6 fewer enucleations.

Currently in the state of Washington, firecrackers, bottle rockets, and missiles are illegal. However, they are legal on a federal level, and thus are legal to purchase and discharge on Indian Reservations. The authors postulate that this is a likely source for many of the devices noted in this study. Even though rockets are illegal in the state of Washington, we show that injuries from bottle rockets are common and that they are highly associated with ocular injuries (Figure 2). In a previous study, the incidence of firework-related ocular injury decreased by $50 \%$ following a ban of bottle rockets and was found to be more effective than public education in reducing the incidence of injury. ${ }^{29}$ Thus, we recommend a similar ban on all public sales of bottle rockets and mortar, the two most common fireworks associated with ocular injuries in our study, not only at a State level but also at the Federal level.

Eye protection has previously been shown to reduce the incidence of ocular injury from fireworks. ${ }^{11}$ Use of appropriate eye-safety equipment may have prevented many of the ocular injuries in our study. Although national labeling rules exist, it is surprising that the mandatory use of eye protection is not mentioned on warning labels for fireworks. We recommend the concurrent sale of eye protection with all firework sales and distribution of protective eyewear at firework displays to decrease the incidence of firework-related ocular trauma. ${ }^{11,29}$

This study has several limitations. First, because of the retrospective nature of the study, case identification was limited to patients listed in the trauma registry, which may not have recorded patients who were treated at our facility and released without admission based on the inclusion criteria. Second, because of our hospital's wide catchment area, follow-up was often limited as many patients travel great distances to our trauma center, and upon acute management of their injuries may choose to transfer their care to providers closer to home or to outpatient providers. Finally, our hospital's level I trauma designation may result in a patient population and injuries that differ in severity from that seen in other treatment facilities.

Ocular injuries from fireworks are common and often visually devastating. Overall, patients in this study experienced improvement in visual acuity after treatment, but a significant proportion of injuries resulted in permanent vision loss. We hope the data presented will increase awareness of these injuries and help to influence public policy and promote further regulation of firework use as well as concomitant selling of appropriate grade-protective eyewear. Safety labels recommending preventative measures such as eye protection may also be helpful in reducing their incidence. Appropriate triage, management, and coordinated treatment by ophthalmologists is critical in the care of these patients in order to ensure the best possible patient outcomes.

\section{Summary}

What was known before

- Firework-related ocular injuries are common; however, previous US studies have not addressed the outcomes, morbidity, and extent of vision loss.

What this study adds

- This study presents the most comprehensive data on outcomes of firework-related ocular injury in the United States and serves to update findings last published over 10 years ago in the United States.

- This study presents visual acuity outcomes of fireworkrelated eye injuries.

- Authors suggest specific ways to decrease the risk of firework-related ocular injuries through evidence presented in these data.

\section{Conflict of interest}

The authors declare no conflict of interest.

\section{Acknowledgements}

We thank Joyce McQuaid for facilitating data retrieval from the University of Washington trauma registry, which she manages, and Leona Ding for assistance with statistical analysis.

\section{References}

1 Tu Y, Granados DV. 2013 Fireworks Annual Report. Fireworks-Related Deaths, Emergency Department-Treated 
Injuries, and Enforcement Activities during 2013. Available at https://www.cpsc.gov//Global/Research-and-Statistics / Injury-Statistics/Fuel-Lighters-and-Fireworks/2013 FireworksReport.pdf (accessed 8 February 2015).

2 Tavakoli H, Khashayar P, Amoli HA, Esfandiari K, Ashegh H, Rezaii J et al. Firework-related injuries in Tehran's Persian Wednesday Eve Festival (Chaharshanbe Soori). J Emerg Med 2011; 40: 340-345.

3 Fireworks Related Injuries- Marion County, Indiana, 1986-1991. 1992. Available at http://www.cdc.gov/mmwr/preview/ mmwrhtml/00017026.htm (accessed 8 February 2015).

4 See LC, Lo SK. Epidemiology of fireworks injuries: the National Electronic Injury Surveillance System, 1980-1989. Ann Emerg Med 1994; 24: 46-50.

5 Wisse RP, Bijlsma WR, Stilma JS. Ocular firework trauma: a systematic review on incidence, severity, outcome and prevention. Br J Ophthalmol 2010; 94: 1586-1591.

6 Sundelin K, Norrsell K. Eye injuries from fireworks in Western Sweden. Acta Ophthalmol Scand 2000; 78: 61-64.

7 Knox FA, Chan WC, Jackson AJ, Foot B, Sharkey JA, McGinnity FG. A British Ophthalmological Surveillance Unit study on serious ocular injuries from fireworks in the UK. Eye (Lond) 2008; 22: 944-947.

8 Kuhn FC, Morris RC, Witherspoon DC, Mann L, Mester V, Módis L et al. Serious fireworks-related eye injuries. Ophthalmic Epidemiol 2000; 7: 139-148.

9 Chan WC, Knox FA, McGinnity FG, Sharkey JA. Serious eye and adnexal injuries from fireworks in Northern Ireland before and after lifting of the firework ban-an ophthalmology unit's experience. Int Ophthalmol 2004; 25: 167-169.

10 Javitt JC, Zhou Z, Willke RJ. Association between vision loss and higher medical care costs in Medicare beneficiaries costs are greater for those with progressive vision loss. Ophthalmology 2007; 114: 238-245.

11 Thygesen J. Ocular injuries caused by fireworks. 25 years of experience with preventive campaigns in Denmark. Acta Ophthalmol Scand 2000; 78: 1-2.

12 Malik A, Bhala S, Arya SK, Sood S, Narang S. Five-year study of ocular injuries due to fireworks in India. Int Ophthalmol 2013; 33: 381-385.

13 Arya SK, Malhotra S, Dhir SP, Sood S. Ocular fireworks injuries. Clinical features and visual outcome. Indian J Ophthalmol 2001; 49: 189-190.

14 Rashid RA, Heidary F, Hussein A, Hitam WH, Rashid RA, Ghani ZA et al. Ocular burns and related injuries due to fireworks during the Aidil Fitri celebration on the East Coast of the Peninsular Malaysia. Burns 2011; 37: 170-173.

15 Sacu S, Ségur-Eltz N, Stenng K, Zehetmayer M. Ocular firework injuries at New Year's eve. Ophthalmologica 2002; 216: 55-59.

16 Singh DV, Sharma YR, Azad RV. Visual outcome after fireworks injuries. J Trauma 2005; 59: 109-111.

17 Jing Y, Yi-qiao X, Yan-ning Y, Ming A, An-huai Y, Lian-hong Z. Clinical analysis of firework-related ocular injuries during Spring Festival 2009. Graefes Arch Clin Exp Ophthalmol 2010; 248: 333-338.

18 Wilson RS. Ocular fireworks injuries and blindness. An analysis of 154 cases and a three-state survey comparing the effectiveness of model law regulation. Ophthalmology 1982; 89: 291-297.

19 Scanlon PH, Foy C, Chen FK. Visual acuity measurement and ocular co-morbidity in diabetic retinopathy screening. Br J Ophthalmol 2008; 92: 775-778.

20 American Pyrotechnics Association Predicts a Banner Year for Consumer Fireworks Sales 2014. Available at http:// www.americanpyro.com/index.php?option $=$ com_content \&view $=$ article\&id $=206$ :pr-apa-predicts-banner-yearfor-consumer-sales\&catid = 20:site-content (accessed 8 February 2015).

21 Hall JR. Fireworks-Related Injuries, Deaths, and Fires in the U. S. Fire Analysis and Research Division, National Fire Protection Association: Quincy, MA, USA, 1999.

22 Chiang YP, Bassi LJ, Javitt JC. Federal budgetary costs of blindness. Milbank Q 1992; 70: 319-340.

23 Directory of State Laws. Available at http://www. americanpyro.com/state-law-directory (accessed 10 June 2016).

24 Vernon SA. Fireworks and the eye. J R Soc Med 1988; 81: 569-571.

25 Ophthalmologist Warn that Fireworks-Related Injury Can Cause Permanent Vision Loss. 2013. Available at http:/ / www.prnewswire.com/news-releases/ophthalmologistswarn-that-fireworks-related-injuries-can-cause-permanentvision-loss-212200781.html (accessed 5 February 2015).

26 McFarland LV, Harris JR, Kobayashi JM, Dicker RC. Risk factors for fireworks-related injury in Washington State. JAMA 1984; 251: 3251-3254.

27 Stilma JS. Prevention of ocular firework injuries. Nepal J Ophthalmol 2012; 4: 3-4.

28 Berger LR, Kalishman S, Rivara FP. Injuries from fireworks. Pediatrics 1985; 75: 877-882.

29 Bull N. Legislation as a tool to prevent firework-related eye injuries. Acta Ophthalmol 2011; 89: e654-e655.

Supplementary Information accompanies this paper on Eye website (http://www.nature.com/eye) 